\title{
Forecasting Portfolio-Value-at-Risk with Nonparametric Lower Tail Dependence Estimates
}

\author{
Karl Friedrich Siburg, Pavel Stoimenov, and \\ Gregor N.F. Weiß
}

Preprint 2013-04

April 2013

Fakultät für Mathematik

Technische Universität Dortmund

Vogelpothsweg 87

44227 Dortmund 



\title{
Forecasting Portfolio-Value-at-Risk with Nonparametric Lower Tail Dependence Estimates
}

\author{
Karl Friedrich Siburg* \\ Fakultät für Mathematik, Technische Universität Dortmund \\ Pavel Stoimenov ${ }^{\dagger}$ \\ Fakultät für Statistik, Technische Universität Dortmund \\ Gregor N.F. Weiß $\beta^{\ddagger}$
}

Wirtschafts- und Sozialwissenschaftliche Fakultät, Technische Universität Dortmund

April 23, 2013

\begin{abstract}
We propose to forecast the Value-at-Risk of bivariate portfolios using copulas which are calibrated on the basis of nonparametric sample estimates of the coefficient of lower tail dependence. We compare our proposed method to a conventional copula-GARCH model where the parameter of a Clayton copula is estimated via Canonical Maximum-Likelihood. The superiority of our proposed model is exemplified by analyzing a data sample of nine different financial portfolios. A comparison of the out-of-sample forecasting accuracy of both models confirms that our model yields economically significantly better Value-at-Risk forecasts than the competing parametric calibration strategy.
\end{abstract}

Keywords: Copula, tail dependence, nonparametric estimation, Value-at-Risk, Canonical Maximum-Likelihood.

JEL Classification Numbers: C53, C58.

\footnotetext{
*Vogelpothsweg 87, D-44227 Dortmund, Germany, telephone: +49 231755 7211, e-mail: karl.f.siburg@ @ath.tudortmund.de.

${ }^{\dagger}$ Address: Vogelpothsweg 78, D-44227 Dortmund, Germany, e-mail: pavel.stoimenov@gmail.com

${ }^{\ddagger}$ Corresponding author: Otto-Hahn-Str. 6a, D-44227 Dortmund, Germany, telephone: +49 231755 4608, telefax: +49 231755 5231, e-mail: gregor.weiss@tu-dortmund.de. Support by the Collaborative Research Center "Statistical Modeling of Nonlinear Dynamic Processes" (SFB 823) of the German Research Foundation (DFG) is gratefully acknowledged.
} 


\section{Introduction}

Following the events of the recent financial crisis, economists and practitioners alike have stressed the need for a more prudent management of financial risks. There now exists a widespread consensus in the empirical literature that the dependence between the returns of financial assets is asymmetric, nonlinear and time-varying (Longin and Solnik, 2001; Ang and Chen, 2002). Consequently, copula models, which allow for a flexible modeling of nonlinear dependence structures, have been advocated in the seminal papers by Embrechts et al. (2002) and Li (2000) as alternatives to correlation-based models. Since then, several studies have found empirical evidence for elliptical copulas yielding only suboptimal Value-at-Risk (VaR) estimates due to their symmetric tail (in-)dependence (Cherubini et al., 2004; Fischer et al., 2009; Weiß, 2011). It is this inadequacy to model the lower tail dependence often found in financial returns that has spurred the search for alternative parametric copula models apart from the Gaussian and Student's t copula.

In this paper, we propose to forecast the VaR of bivariate portfolios using copulas which are calibrated solely on the basis of nonparametric sample estimates of the coefficient of lower tail dependence (LTD). A first step in this direction has been made in Weiß (2011) and Jäschke et al. (2012) where it is shown that choosing a model based on usual goodness-of-fit tests (see, e.g., Trede and Savu, 2008, for a discussion of copula goodness-of-fit tests) may not provide a good model for tail dependence. In the present work, we go a step further and show that calibrating a copula model by fitting the lower tail dependence (LTD) to the data does indeed yield better VaR forecasts. To fully capture the LTD in the returns on financial assets, we first use the nonparametric estimator of tail dependence proposed by Schmidt and Stadtmüller (2006) and estimate the LTD in a given in-sample of portfolio returns. The estimates for the LTD are then transformed into corresponding parameters of the lower tail dependent Clayton copula. In contrast to estimation procedures based on maximizing the copula's likelihood function, which estimate the copula parameters by using information on the complete dependence structure, we calibrate the model by only incorporating information on the tail dependence in the data. Our approach is motivated by the notion that the knowledge of the tails as well as the center of a distribution will be merged by the ML-estimation 
into one single parameter of an Archimedean copula. As a consequence, forecasting risk measures in the lower left tail in the distribution will lose in accuracy as the tails are not adequately modeled. After calibrating the Clayton copula with the nonparametric estimate of LTD, we forecast the VaR of a portfolio using GARCH models for the marginals. ${ }^{1}$

We compare our proposed method to a conventional copula-GARCH model where the parameter of the Clayton copula is estimated via Canonical Maximum-Likelihood (CML) (Kim et al., 2007). We examine the usefulness of our method by analyzing a data sample of nine different portfolios consisting of different financial assets. To be precise, we model the distributions of the returns on one electricity index, one natural gas index, two commodities as well as the stocks of five electric utility service providers. ${ }^{2}$ Our choice of financial assets is guided by the wish to test the forecasting accuracy of our proposed model based on a data sample that includes sufficient tail events. Consequently, we model the distribution of securities that presumably were heavily influenced by the financial crisis as well as the Fukushima Daiichi nuclear disaster in Japan.

The results presented in this paper show that our proposed method of calibrating a copula model with a nonparametric estimate of the lower tail dependence performs significantly better than the standard method of estimating the copula parameter via Canonical Maximum Likelihood with respect to risk forecasting. We show in formal backtesting that our calibration strategy yields significantly fewer VaR-exceedances than the parametric benchmark.

The remainder of this article is structured as follows. Section 2 introduces the nonparametric estimator of the coefficient of lower tail dependence as well as the copula-GARCH model we use. In Section 3 the results of the empirical study are presented and discussed. Concluding remarks are given in Section 4.

\footnotetext{
${ }^{1}$ Thus yielding the standard copula-GARCH approach to estimating and forecasting multivariate portfolio VaR (Hsu et al., 2012; Nikoloulopoulos et al., 2011; Weiß, 2011).

${ }^{2}$ For a related study of the dependence structures inherent in energy commodities, see Börger et al. (2009).
} 


\section{Methodology}

\subsection{Copulas and Tail Dependence Functions}

The theory of copulas dates back to Sklar (1959). A copula is a function that embodies all the information about the dependence structure between the components of a random vector. From a probabilistic point of view, it is a multivariate distribution function with uniformly distributed margins on the interval $[0,1]$. Let us consider two random variables $X, Y$ with continuous marginal distribution functions $F(x):=P(X \leq x)$ and $G(y):=P(Y \leq y)$, respectively, where $x$ and $y$ are real numbers. By Sklar's theorem, there exists a unique copula $C$ such that

$$
P(X \leq x, Y \leq y)=C(F(x), G(y))
$$

Conversely, if $C$ is a copula and $F, G$ are distribution functions, then the function defined via (1) is a bivariate distribution function with margins $F, G$. It follows that copulas can be interpreted as dependence functions since they separate the marginal distributions from the dependence structure. We have

$$
C(u, v)=P(U \leq u, V \leq v)=P\left(X \leq F^{-1}(u), Y \leq G^{-1}(v)\right)
$$

for all $u, v \in[0,1]$, where $F^{-1}$ and $G^{-1}$ denote the (generalized) inverses of $F$ and $G$, respectively.

Let $\bar{F}(x):=1-F(x)=P(X>x)$ and $\bar{G}(y):=1-G(y)=P(Y>y)$ denote the corresponding survival functions of $X$ and $Y$. Define a function $\hat{C}$ by

$$
\hat{C}(u, v)=u+v-1+C(1-u, 1-v)
$$

for all $u, v \in[0,1]$. Then we obtain

$$
P(X>x, Y>y)=\hat{C}(\bar{F}(x), \bar{G}(y))
$$

The function $\hat{C}$ is a copula itself and is called the survival copula of $X$ and $Y$. In view of (4), the 
survival copula links the joint survival function to its univariate margins in a manner completely analogous to the one in which the copula connects the joint distribution function to its margins. Hence, for all $u, v \in[0,1]$, we have

$$
\begin{aligned}
\hat{C}(u, v) & =P(U>1-u, V>1-v) \\
& =P\left(X>F^{-1}(1-u), Y>G^{-1}(1-v)\right) .
\end{aligned}
$$

For further details regarding the theory of copulas we refer the reader to Nelsen (2006).

As the focus of this paper is to characterize and measure extreme dependence, the rest of this section is devoted to the concept of tail dependence, which concentrates on the upper and lower quadrant tails of the joint distribution. The standard way to determine whether $X$ and $Y$ are tail dependent is to look at the so-called lower and upper tail dependence coefficients, denoted by LTD and UTD, respectively. LTD is the limit (if it exists) of the conditional probability that $X$ is less than or equal to the $u$-th quantile of $F$, given that $Y$ is less than or equal to the $u$-th quantile of $G$ as $u$ approaches 0 , i.e.

$$
\mathrm{LTD}:=\lim _{u \rightarrow 0^{+}} P\left(X \leq F^{-1}(u) \mid Y \leq G^{-1}(u)\right) .
$$

Similarly, UTD is the limit (if it exists) of the conditional probability that $X$ is greater than the $u$-th quantile of $F$, given that $Y$ is greater than the $u$-th quantile of $G$ as $u$ approaches 1 , i.e.

$$
\mathrm{UTD}:=\lim _{u \rightarrow 1^{-}} P\left(X>F^{-1}(u) \mid Y>G^{-1}(u)\right) .
$$

From a practitioner's point of view, lower tail dependence can be interpreted as the limiting likelihood of two financial assets to crash together. Consequently, several studies in economics have underlined the necessity for econometric models to account for the possibility of lower tail dependent assets in financial portfolios (see, e.g., Poon et al., 2004). While many empirical studies like, e.g., Christoffersen et al. (2012) use parametric models for describing tail dependent financial 
data, it is interesting to note that the tail dependence coefficients are nonparametric and depend only on the copula $C$ of $X$ and $Y$. In particular, we have

$$
\mathrm{LTD}=\lim _{u \rightarrow 0^{+}} \frac{C(u, u)}{u}
$$

and

$$
\mathrm{UTD}=\lim _{u \rightarrow 1^{-}} \frac{1-2 u+C(u, u)}{1-u}=\lim _{u \rightarrow 0^{+}} \frac{\hat{C}(u, u)}{u}
$$

where $\hat{C}$ is the survival copula of $X$ and $Y$ defined in (3). In view of these identities, passing from LTD to UTD is the same as passing from $C$ to $\hat{C}$ so we may, and will, concentrate on LTD and disregard UTD for the rest of our paper. ${ }^{3}$

\subsection{Nonparametric Estimation of Tail Dependence}

To estimate the lower tail dependence coefficient, we will use the estimator for LTD introduced in Schmidt and Stadtmüller (2006). Let $(X, Y)$ be a bivariate random vector with distribution function $F$ and copula $C$, having marginal distributions functions $G$ and $H$, respectively, and $\left(X^{(1)}, Y^{(1)}\right), \ldots,\left(X^{(T)}, Y^{(T)}\right)$ be an independent and identically distributed sample of $(X, Y)$ of size $T$. If we denote the empirical distribution functions corresponding to $F, G, H$ by $F_{T}, G_{T}, H_{T}$, respectively, the empirical copula $C_{T}$ is given by

$$
C_{T}(u, v)=F_{T}\left(G_{T}^{-1}(u), H_{T}^{-1}(v)\right)
$$

In this setting, the estimator introduced in Schmidt and Stadtmüller (2006) is defined as

$$
\widehat{\operatorname{LTD}}_{T}:=\frac{T}{k} C_{T}\left(\frac{k}{T}, \frac{k}{T}\right)
$$

\footnotetext{
${ }^{3}$ In the context of modeling the profit and loss distributions of financial assets, LTD is usually of much larger interest to investors and risk managers than UTD.
} 
where $k=k(T)$ is some parameter satisfying $k \rightarrow \infty$ and $k / T \rightarrow 0$ as $T \rightarrow \infty$. To compute the nonparametric LTD estimate, consider the ranks $R_{T 1}^{(t)}$ and $R_{T 2}^{(t)}(t=1, \ldots, T)$ of the observations $X^{(t)}$ and $Y^{(t)}$ in the sample. The LTD estimate is then given by

$$
\left.\widehat{\operatorname{LTD}}_{T}=\frac{1}{k} \sum_{t=1}^{T} \mathbf{1}_{\left\{R_{T 1}^{(t)} \leq k\right.} \text { and } R_{T 2}^{(t)} \leq k\right\}
$$

where the parameter $k \in\{1, \ldots, T\}$ is chosen by the use of a plateau-finding algorithm.

\subsection{Value-at-Risk Forecasting}

In this subsection, we quickly review the modeling of the marginals as well as the procedure to forecast portfolio Value-at-Risk.

Financial data are commonly found to exhibit conditional heteroscedasticity, skewness and leptokurtosis in logarithmic returns. As most theoretical results for copulas including Sklar's theorem only hold for i.i.d. samples, financial data are usually filtered using univariate GARCH(1,1) models to yield approximately i.i.d. samples of standardized residuals. ${ }^{4}$ We follow the vast majority of studies in the financial econometrics literature on copula models (Jondeau and Rockinger, 2006; Fantazzini, 2009; Hafner and Reznikova, 2010) and employ standard GARCH(1,1) models with Student's t-distributed innovations to filter our initial data sample. ${ }^{5}$

We consider time series of daily mid prices $P_{t}(t=0,1, \ldots, T)$ of a financial asset. The $\operatorname{logarithmic~return} R_{t}$ on the asset on day $t$ is defined as $R_{t}:=\log \left(P_{t} / P_{t-1}\right)$. Our main focus lies on the modeling of the joint distribution of the return on a portfolio of 2 assets with returns $\left(R_{t 1}, R_{t 2}\right)$. The marginal distributions of this random vector are modeled using $\mathrm{GARCH}(1,1)$ specifications

$$
\begin{aligned}
R_{t j} & =\mu_{j}+\sigma_{t j} Z_{t j}, \\
\sigma_{t j}^{2} & =\alpha_{0 j}+\alpha_{1 j} R_{t-1, j}^{2}+\beta_{j} \sigma_{t-1, j}^{2}, j=1, \ldots, d ; t=1, \ldots, T,
\end{aligned}
$$

\footnotetext{
${ }^{4}$ Garmann and Grundke (2013) show for copula goodness-of-fit tests that using GARCH filters to account for the time-varying volatility in financial returns is indeed necessary when later estimating a copula model.

${ }^{5}$ Hansen and Lunde (2005) show that the GARCH(1,1) specification, in general, cannot be outperformed by more complex variants of the ARCH or GARCH models.
} 
where $Z_{t j}$ are Student's t-distributed innovations. The vectors $\mathbf{Z}_{t}=\left(Z_{t 1}, Z_{t 2}\right)$ with $t=1, \ldots, T$ are assumed to be distributed according to

$$
F_{\mathbf{Z}}\left(\mathbf{z} ; \nu_{1}, \nu_{2}, \omega\right)=C\left[F_{1}\left(z_{1} ; \nu_{1}\right), F_{2}\left(z_{2} ; \nu_{2}\right) ; \omega\right]
$$

with parameters $\nu_{1}, \nu_{2}$ for the innovations' distribution and a copula with parameter $\omega$. As the dependence in financial market data is commonly characterized by strong lower tail dependence, we only consider the Clayton copula as a parametric family for $C{ }^{6}$ The parameters of the marginal models are estimated via Maximum-Likelihood. For the parameter of the copula $C$, two different estimation procedures will be used. First, the parameter of the copula will be estimated by using the standard Canonical Maximum-Likelihood (CML) method with rank-transformed pseudoobservations (McNeil et al., 2005). ${ }^{7}$ Second, we alternatively obtain the copula parameter by estimating the coefficient of lower tail dependence nonparametrically with the estimator of Schmidt and Stadtmüller (2006) and converting this nonparametric estimate into the (uniquely identified) parameter of the Clayton copula.

In the following as well as in our empirical study, we consider equally-weighted portfolios consisting of two financial assets with log returns $\left(R_{t 1}\right)$ and $\left(R_{t 2}\right)$. Using an in-sample of $T$ observations (i.e., trading days), we then wish to forecast the Value-at-Risk and Expected Shortfall of the portfolio for the following day $T+1$ via Monte Carlo simulation. The algorithm we use for forecasting the VaR and ES is an adapted version of the procedure laid out by Nikoloulopoulos et al. (2011). Their algorithm, however, only considers an in-sample estimation whereas we employ an out-of-sample version of their original simulation procedure extended by Weiß (forthcoming). Then, the VaR and ES are forecasted as follows:

\footnotetext{
${ }^{6}$ Other parametric copula families are obviously possible and more flexible approaches like mixture copulas could yield an even better fit of the model to the data. The focus of our paper, however, lies on an accurate modeling of the lower tail dependence in financial market data. We therefore opted for a simple Clayton copula as our dependence model as the coefficient of lower tail dependence is easily transformed into the parameter of the Clayton copula.

${ }^{7}$ Note that the CML estimator has been shown to yield less biased parameter estimates than, e.g., the Inference For Margins or Full Maximum-Likelihood methods (Kim et al., 2007). It has since been accepted as the method of choice for estimating copula parameters (Genest et al., 2009).
} 
- For $K=5,000$, simulate $K$ observations $u_{T+1,1}^{(k)}, u_{T+1,2}^{(k)}(k=1, \ldots, K)$ from the fitted copula.

- Convert $u_{T+1, j}^{(k)}$ to $z_{T+1, j}^{(k)}(j=1,2)$ using the quantile function of the Student's t-distribution.

- Transform $z_{T+1, j}^{(k)}$ into the simulated return $R_{T+1, j}^{(k)}=\hat{\mu}_{j}+\hat{\sigma}_{T+1, j} z_{T+1, j}^{(k)}$ where $\hat{\sigma}_{T+1, j}$ and $\hat{\mu}_{j}$ $(j=1,2)$ are the forecasted one-step ahead conditional volatility and mean values from the previously fitted marginal models.

- Compute the simulated portfolio return as $R_{T+1, p}^{(k)}=2^{-1} \sum_{j=1}^{2} R_{T+1, j}^{(k)}$.

- Smooth the simulated log returns by kernel density estimation (Epanechnikov kernel) (Pritsker, 2006; Alexander and Sheedy, 2008).

- Compute the VaR at the $100(1-\alpha) \%$ confidence level for day $T+1$ as the $\alpha$-quantile of the kernel density estimate.

- Update the information set with the actual portfolio return $R_{T+1, p}$, reestimate all models and forecast the portfolio return for day $T+2$ and so forth.

The procedure described above is repeated for both the copula model based on the CML parameter estimate as well as the parameter estimate obtained by nonparametrically estimating the coefficient of lower tail dependence.

The resulting time series of VaR-exceedances, i.e., the days on which the portfolio suffered losses exceeding the VaR forecast on that day, are backtested by the use of the test of conditional coverage (Christoffersen, 1998; Christoffersen and Pelletier, 2004). The p-values of the test are approximated via parametric bootstrapping. ${ }^{8}$

After forecasting the VaR for each day in the out-of-sample, we additionally compute the estimate for the portfolios' sample Expected Shortfall as the mean of the simulated returns beyond the

\footnotetext{
${ }^{8}$ Additionally, the forecasting performance of the models could also be analyzed by the ranking model proposed by Şener et al. (2012). As we only consider two models, however, such a ranking would yield only a marginal incremental insight into the forecasting accuracy of the models.
} 
estimated VaRs in the out-of-sample. The models' Expected Shortfall estimates are then backtested using the sample asymptotics derived by Wong (2008) under a standard normal null hypothesis.

\section{Empirical Study}

\subsection{Data}

Our sample includes the log returns on one electricity index (ICE UK Electricity Baseload Index), one natural gas index (EEX EGIX NCG Index), two commodities (Crude Oil - Brent Dated FOB and MLCX - South African Coal) and the stocks of five global electric utility service providers (BP, E.ON, Royal Dutch Shell, RWE and Tohoku Electric Power). The data were obtained from Thomson Reuters Datastream. ${ }^{9}$ The complete sample covers the period from May 12, 2008 to June 3, 2011 and includes 800 trading days. Our sample thus includes both the peak of the recent financial crisis as well as the Fukushima Daiichi nuclear disaster in Japan (March 2011). Time series plots of the data used in our empirical study are presented in Figure 1.

— insert Figure 1 here —

The plots in Figure 1 highlight several distinct challenges for the econometric forecasting of risk measures for portfolios built from these assets. First, we can see from the plots that the different assets are characterized by extremely different levels of overall volatility as well as price movements (see, e.g., the extreme spikes in the ICE index at the beginning of our sample as compared to the relatively calm period following this volatility cluster). For all nine time series, we can observe increased levels of volatility in the returns during the first 250 trading days in the in-sample coinciding with the climax of the financial crisis. The Fukushima Daiichi nuclear disaster at the end of our sample seems to have caused extreme tail events in the stock returns of Tohoku Electric Power. At the same time, both E.ON and Shell experienced only moderate losses on their stocks.

\footnotetext{
${ }^{9}$ Ince and Porter (2006) propose several screening procedures for stock prices obtained from Thomson Reuters Datastream. We control our sample both for stock prices below $\$ 1$ (which could lead to erroneous log returns due to Datastream's practice of rounding prices) and log returns above $300 \%$ that are reversed within one month. None of our time series suffer from these known data errors in Datastream.
} 
While BP also did not experience any downside movements of its stock during the Fukushima Daiichi disaster, the stock nevertheless suffered severe losses between April and July 2010 following the Deepwater Horizon oil spill (sample days 508 to 559). Finally, the plots reveal that both the initial in- as well as the out-of-samples for the nine assets we consider differ considerably with respect to the returns' volatility and tail events. While for some assets return volatility is low in the initial in-sample and increases thereafter (see, e.g., the plot for Tohoku Electric Power), the opposite (i.e., lower asset return volatilities in the out-of-sample) is true for several other time series (see, e.g., E.ON, BP and Shell).

Descriptive statistics as well as results of the Jarque-Bera test of normally distributed log returns are given in Table 1.

— insert Table 1 here -

The results given in Table 1 underline our first impression of the time series plots that several of the assets used in our study experienced extreme tail events both in our in-sample as well as the out-of-sample. For example, the ICE UK Electricity index exhibited a minimum log return of $-69.31 \%$ in the in-sample while its minimum in the out-of-sample was just $-5.35 \%$. Conversely, both RWE and Tohoku Electric Power had modest minimum log returns of $-8.47 \%$ and $-9.45 \%$ in the in-sample but suffered extreme minimum returns of $-14.04 \%$ and $-23.84 \%$ in the outof-sample, respectively. Unreported results on the skewness and kurtosis of the data confirm the stylized facts of skewed and fat-tailed returns in our full sample. As a consequence, the JarqueBera test of normally distributed log returns can be rejected for all time series in the in-sample. Although the data for two time series (MLCX and Royal Dutch Shell) seem to be approximately normally distributed in the out-of-sample, we nevertheless employ a $\operatorname{GARCH}(1,1)$ model with Student's t-distributed innovations as the Jarque-Bera test is rejected for both series in the initial in-sample. Also note that the volatility of the time series differs significantly both across the nine assets we use as well as across time.

From the nine assets, we build nine bivariate portfolios for which we forecast the portfolio Value-at-Risk on each day of the out-of-sample containing 300 trading days. The portfolios we 
consider are built as follows: ICE UK Electricity Baseload and EEX EGIX NCG Index (portfolio 1), Crude Oil Brent and MLCX (portfolio 2), Crude Oil Brent and E.ON (portfolio 3), Crude Oil Brent and Shell (portfolio 4), Crude Oil Brent and BP (portfolio 5), RWE and E.ON (portfolio 6), RWE and Shell (portfolio 7), RWE and BP (portfolio 8) and Shell and Tohoku Electric Power (portfolio 9). Both assets enter each portfolio with equal weights to control for a possibly biasing influence the specific choice of weights might have on the forecasting accuracy of our models.

\subsection{Results}

We start the discussion of the results of our empirical study by first comparing the parametric and nonparametric estimates of the coefficient of lower tail dependence. The parametric and nonparametric estimates of the LTD for all nine bivariate portfolios in the out-of-sample are presented in Figure 2.

— insert Figure 2 here -

Across all nine portfolios, we can observe a general trend of the parametric model to yield lower LTD estimates than the nonparametric estimator and thus possibly underestimate the true level of lower tail dependence between the two portfolio constituents. With the exception of portfolios five and six (Crude Oil Brent/BP and RWE/E.ON) and few forecasting days for portfolios four and eight (Crude Oil Brent/Shell and RWE/BP), the parametric estimates of the lower tail dependence are always lower than the corresponding nonparametric estimates. For portfolios one and three (ICE/EEX EGIX NCG and Crude Oil Brent/E.ON), both the time series of the paramet-

ric and nonparametric LTD estimates seem to comove with the difference between the two staying constant for any given day in the out-of-sample. In contrast, estimates for portfolios seven and eight (RWE/Shell and RWE/BP) differed only slightly at the beginning of the out-of-sample but dispersed considerably afterwards. For portfolio nine (Shell/Tohoku), the parametric model yields LTD estimates that are constantly zero due to an overall bad fit of the parametric Clayton copula model. Only for portfolio six (RWE/E.ON) does the nonparametric LTD estimator yield lower 
LTD estimates than the parametric model via Canonical Maximum Likelihood estimation. The plots given in Figure 2 thus confirm our hypothesis that the LTD estimates extracted from the parametric models fitted via CML and the nonparametric estimates differ considerably. It thus remains to be seen whether these differences in the way the LTD in a portfolio is estimated has a significant influence on the accuracy of Value-at-Risk forecasts for the portfolio.

To test our hypothesis that copula models calibrated by the use of nonparametric estimates of lower tail dependence can significantly improve the accuracy of VaR forecasts in comparison to a parametric model calibrated via Maximum Likelihood, we use both model approaches to forecast the VaR and ES for the nine portfolios described above for each day in our out-of-sample consisting of 300 trading days. Following the general procedure laid out in Section 2.3, we compare the VaR and ES estimates from two different model setups.

The first model we consider is a Clayton copula model with $\operatorname{GARCH}(1,1)$ marginals (tdistributed innovations) and the parameter of the Clayton copula being estimated via Canonical Maximum Likelihood (parametric model). The second model we consider equals the first one with the exception that the parameter of the Clayton copula is estimated via the transformed nonparametric estimate of the coefficient of lower tail dependence (nonparametric model). Both models are estimated based on samples of size 500. We use both fitted models to forecast the portfolio returns, Value-at-Risk as well as Expected Shortfall for the following trading day. The estimation sample is then updated with the next day in the out-of-sample and all models are reestimated using the updated sample. The procedure is repeated for all 300 trading days in the out-of-sample and the resulting VaR and ES estimates are backtested using the formal tests of Christoffersen and Pelletier (2004) and Wong (2008). The confidence level for the VaR forecasting is $95 \%$. For each day in the out-of-sample, the VaR and ES forecasts are computed based on 5,000 simulated daily portfolio returns. The resulting VaR forecasts from the two models as well as the realized portfolio returns for all nine portfolios are shown in Figure 3.

— insert Figure 3 here - 
The plots given in Figure 3 provide us with a first hint at the superiority of our proposed model based on nonparametric LTD estimates. With the exception of portfolio one (ICE/EEX EGIX), the model based on the CML parameter estimates seems to underestimate risk for all remaining eight portfolios compared to the approach based on nonparametric LTD estimates. The reason for the considerably bad fit of both models for portfolio one is easily seen from the time series plot of the ICE index in Figure 1 which shows that the initial in-samples of the portfolio are characterized by extreme volatility in the log returns that vanishes completely after about 70 trading days. Consequently, the VaR forecasts computed from in-samples containing these highly volatile times are too conservative. ${ }^{10}$ For the remaining eight portfolios, both models seem to yield adequate VaR forecasts with the VaR forecasts based on the nonparametric LTD estimates being slightly smaller than the corresponding forecasts from the model estimated via CML.

To formally test the accuracy of the VaR and ES forecasts of both models, we performe both the test of conditional coverage (CC test) by Christoffersen and Pelletier (2004) and the Expected Shortfall (ES) backtest proposed by Wong (2008) on the two models' results. The results for the tests are presented in Table 2. The table presents the number of VaR-exceedances, the p-value of the test of conditional coverage as well as the test decision for the ES backtest for all nine portfolios and both models (parametric and nonparametric) used for forecasting the portfolio VaR and ES. The expected number of VaR-exceedances for each portfolio is 15.

— insert Table 2 here -

The results in Table 2 confirm our finding that the VaR forecasts based on CML parameter estimates significantly underestimate portfolio risk. The number of VaR-exceedances is significantly higher for the parametric model than for the forecasts computed by using the nonparametric LTD estimates. Only for portfolio one (ICE/EEX EGIX) does the nonparametric modeling of the lower tail dependence not improve the accuracy of the VaR forecasts. However, this result is most likely

\footnotetext{
${ }^{10}$ Note that both models are capable of adapting quickly to extreme portfolio returns as can be seen from the plot for portfolio nine (Shell/Tohoku). Although both models are not able to anticipate the extreme negative return around trading day 240 (i.e., the Fukushima Daiichi nuclear disaster), VaR forecasts immediately drop down to about $-13 \%$ just five trading days after the extreme shock.
} 
due to the highly irregular time series behaviour of the ICE index (extremely high volatility in the beginning compared to virtually no price movements in the later parts of the sample). In contrast, the parametric model yielded $19 \mathrm{VaR}$-exceedances more than expected for portfolios three and four. While this result alone is indicative of an inaccurate forecasting of portfolio risk, we cannot decide per se whether the number of VaR-exceedances is still acceptable or not. To this end, the p-values for the test of conditional coverage reveal that for four portfolios (three, four, six and seven), the VaR forecasts of the model using the CML parameter estimates are rejected while the model based on the nonparametric LTD estimates yields acceptable VaR forecasts. For portfolios two, five, eight and nine, the parametric model yields considerably more VaR-exceedances which, however, cannot be rejected by the formal backtest. In summary, our proposed method of calibrating a copula model with a nonparametric estimate of the lower tail dependence with the purpose of forecasting a quantile-based risk measure in the lower tail of a portfolio's distribution performs significantly better than the standard method of estimating the copula parameter via Canonical Maximum Likelihood. The backtesting results underline the finding that the differences between the two models' VaR forecasts are highly significant.

Conversely, the formal ES backtest does not reject any of the models' ES forecasts with the exception of the nonparametric model's forecasts for portfolio five (Crude Oil Brent/BP). It thus seems that the ES forecasts from both models do not differ considerably. The results on the ES backtests have to be interpreted with care, however, as the sample size for the test was regularly smaller than ten. ${ }^{11}$

\section{Conclusion}

This paper proposes to forecast the VaR of bivariate portfolios by the use of copula models which are calibrated with nonparametric estimates of the coefficient of lower tail dependence. Our approach is motivated by the notion that copula models that are estimated based on information on

\footnotetext{
${ }^{11}$ Backtesting forecasts of Expected Shortfall is known to suffer from small sample biases as noted, e.g., by Wong (2008).
} 
the complete underlying distribution could significantly underestimate tail risk. Using the Canonical Maximum-Likelihood estimator as a benchmark, we show that the ML-estimated model yields significantly smaller estimates of the coefficient of lower tail dependence than our proposed model. These results hint at the possibility that a Clayton copula estimated via Maximum-Likelihood could systematically underestimate the lower tail dependence inherent in a given data sample.

The results of our empirical study strongly corroborate this finding as we find our proposed model to perform significantly better in VaR backtesting than the ML-estimated benchmark. Our proposed model produces fewer VaR-exceedances in out-of-sample backtesting than the benchmark for all nine portfolios we consider. For four out of these nine bivariate portfolios, we find that the differences in the number of VaR-exceedances is statistically significant as evidenced by the formal test of conditional coverage.

It could be argued that this paper's focus on bivariate copulas is a serious limitation of our work's pratical relevance. Although our proposed model significantly improves bivariate portfolio VaR forecasts, portfolios of financial assets are often of much higher dimension. We nevertheless believe our findings from the analysis of bivariate copulas to be highly relevant. Recent work by Aas et al. (2009) and Dissmann et al. (2013) emphasizes that so-called pair-copula constructions are extremely well suited for modeling financial returns in high dimensions. As these hierarchical models are built from bivariate copulas as buildings blocks, our findings should be directly applicable to high-dimensional pair-copula models. The extension of our work to the field of pair-copula modeling thus appears to be a promising avenue for further research.

\section{Acknowledgements}

Support by the Collaborative Research Center "Statistical Modeling of Nonlinear Dynamic Processes" (SFB 823) of the German Research Foundation (DFG) is gratefully acknowledged. 


\section{References}

Aas, K., C. Czado, A. Frigessi, And H. Bakken (2009): "Pair-Copula Constructions of Multiple Dependence," Insur Math Econ, 44, 182-198.

Alexander, C. AND E. Sheedy (2008): "Developing a stress testing framework based on market risk models," J Bank Financ, 32, 2220-2236.

ANG, A. AND J. CHEN (2002): "Asymmetric correlations of equity portfolios," J Financ Econ, 63, 443-494.

Börger, R., A. Cartea, R. Kiesel, And G. Schindlmayr (2009): "A mulitvariate commodity analysis and applications to risk management," J Future Mark, 29(3), 197-217.

Cherubini, U., E. Luciano, And W. Vecchiato (2004): Copula Methods in Finance, John Wiley and Sons.

Christoffersen, P. (1998): “Evaluating Interval Forecasts,” Int Econ Rev, 39, 841-862.

Christoffersen, P., V. Errunza, K. Jacobs, And H. LAnglois (2012): "Is the Potential for International Diversification Disappearing? A Dynamic Copula Approach," Rev Financ Stud, 25(12), 3711-3751.

Christoffersen, P. And D. Pelletier (2004): "Backtesting Value-at-Risk: A DurationBased Approach," J Financ Econom, 2, 84-108.

ŞEner, E., S. BARonyan, And L. A. MengütÜRK (2012): "Ranking the predictive performances of value-at-risk estimation methods," Int J Forecasting, 28, 849-873.

Dissmann, J., E. Brechmann, C. Czado, And D. Kurowicka (2013): "Selecting and Estimating Regular Vine Copulae and Application to Financial Returns," Comput Stat Data Anal, 59(1), 52-69.

Embrechts, P., A. McNeil, And D. Straumann (2002): "Correlation and dependence in risk management: properties and pitfalls," in Risk Management: Value at Risk and Beyond, ed. by M. Dempster, Cambridge University Press, Cambridge, 176-223.

FAntazZIni, D. (2009): "The Effects of Misspecified Marginals and Copulas on Computing the Value at Risk: A Monte Carlo Study," Comput Stat Data Anal, 53, 2168-2188.

Fischer, M., C. KÖCK, S. SchlÜter, AND F. Weigert (2009): “An empirical analysis of multivariate copula model," Quant Financ, 7, 839-854.

GARMANN, S. AND P. GRUNDKE (2013): "On the influence of autocorrelation and GARCHeffects on goodness-of-fit tests for copulas," Eur J Financ, 19(1), 75-88.

Genest, C., B. RÉmillard, And D. Beaudoin (2009): "Goodness-of-fit tests for copulas: A review and a power study," Insur Math Econ, 44, 199-213. 
HAFNER, C. AND O. REZNiKOVA (2010): "Efficient estimation of a semiparametric dynamic copula models," Comput Stat Data Anal, 54(11), 2069-2627.

Hansen, P. And A. Lunde (2005): “A Forecast Comparison of Volatility Models: Does Anything Beat a GARCH(1,1)?” J Appl Econometr, 20, 873-889.

Hsu, C., C. Huang, And W. Chiou (2012): "Effectiveness of Copula-Extreme Value Theory in Estimating Value-at-Risk: Empirical Evidence from Asian Emerging Markets," Rev Quant Financ Account, 39(4), 447-468.

InCE, O. AND R. Porter (2006): "Individual Equity Return Data From Thomson Datastream: Handle With Care!" J Financ Res, 29, 463-479.

JäschKe, S., K. SiburG, And P. Stoimenov (2012): "Modeling dependence of extreme events in energy markets using tail copulas," J Energy Mark, 5, 63-80.

JONDEAU, E. AND M. RoCKINGER (2006): “The copula-GARCH model of conditional dependencies: An international stock market application," J Int Money Financ, 25(5), 827-853.

Kim, G., M. Silvapulle, And P. Silvapulle (2007): "Comparison of semiparametric and parametric methods for estimating copulas," Comput Stat Data Anal, 51, 2836-2850.

LI, D. (2000): “On Default Correlation: A Copula Function Approach,” J Fixed Income, 9, 43-54.

LONGIN, F. AND B. SOLNIK (2001): "Extreme correlation of international equity," J Financ, 56, 649-676.

McNeil, A., R. Frey, And P. Embrechts (2005): Quantitative Risk Management, Princeton University Press, Princeton.

Nelsen, R. (2006): An Introduction to Copulas, Springer, second ed.

Nikoloulopoulos, A., H. Joe, And H. Li (2011): "Vine copulas with asymmetric tail dependence and applications to financial return data," Comput Stat Data Anal, forthcoming.

Poon, S.-H., M. Rockinger, And J. TAWn (2004): "Extreme Value Dependence in Financial Markets: Diagnostics, Models and Financial Implications," Rev Financ Stud, 17(2), 581-610.

PRITSKER, M. (2006): “The hidden dangers of historical simulation,” J Bank Financ, 30, 561582.

SCHMIDT, R. AND U. STADTMÜLlER (2006): "Nonparametric estimation of tail dependence," Scand J Stat, 33, 307-335.

SKLAR, A. (1959): "Fonctions de répartition à $n$ dimension et leurs marges," Publications de l'Institut de Statistique de L'Université de Paris, 8, 229-231.

Trede, M. AND C. SAVU (2008): “Goodness-of-fit Tests for Parametric Families of Archimedean Copulas," Quant Financ, 8, 109-116. 
WEISS, G. (2011): "Are Copula-GoF-tests of any practical use? Empirical evidence for stocks, commodities and FX futures," $Q$ Rev Econ Financ, 51(2), 173-188.

(forthcoming): "Copula-GARCH versus dynamic conditional correlation: an empirical study on VaR and ES forecasting accuracy," Rev Quant Financ Account, doi:10.1007/s11156012-0311-2.

WONG, W. (2008): "Backtesting trading risk of commercial banks using expected shortfall," $J$ Bank Financ, 32, 1404-1415. 
Figures and Tables 

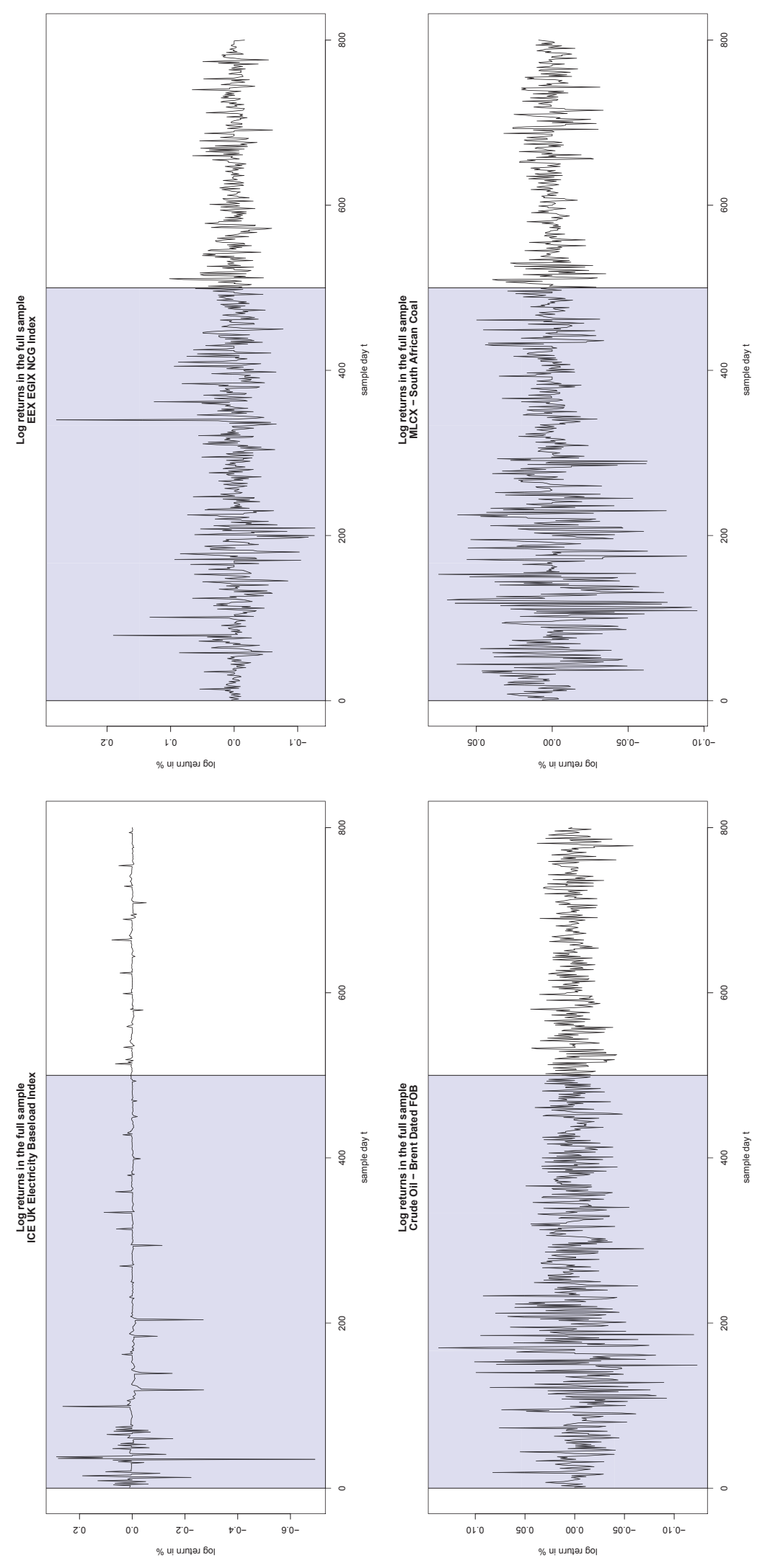

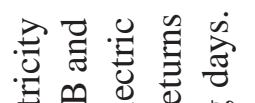
등 कै

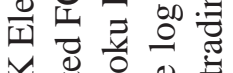
光

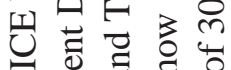

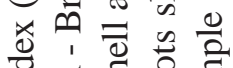
宁的음

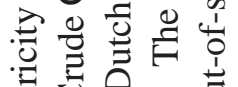

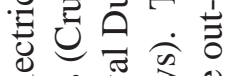
语 跣

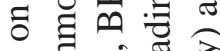

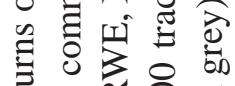
政 \& on $z$ 要

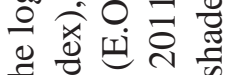

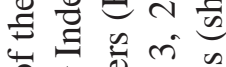

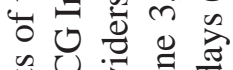

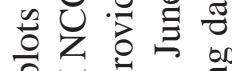

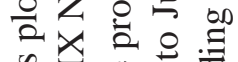

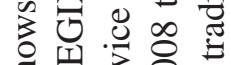

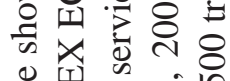

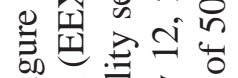

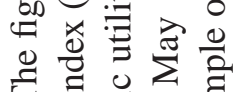
. ह ह

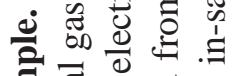

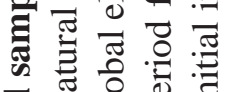
西 $\frac{\mathrm{O}}{50} \mathrm{0}$

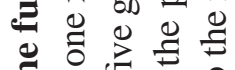

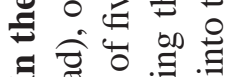

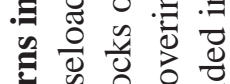

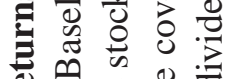
๘ on 항

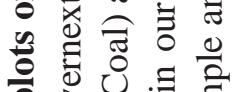
흘 $0 . \Xi$

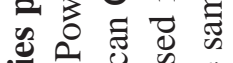
눌 部安

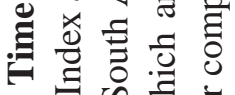
范的言

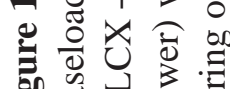

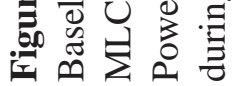



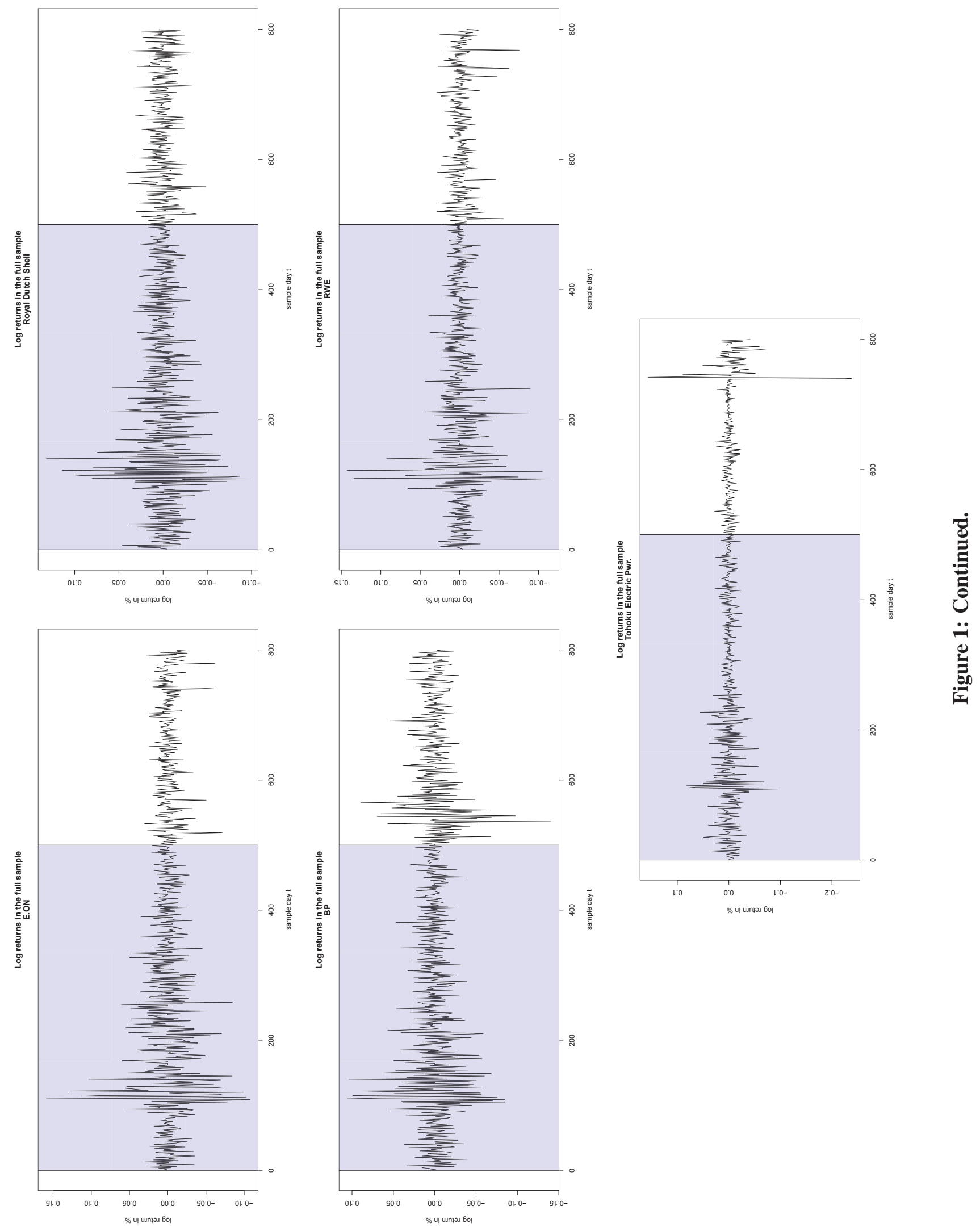

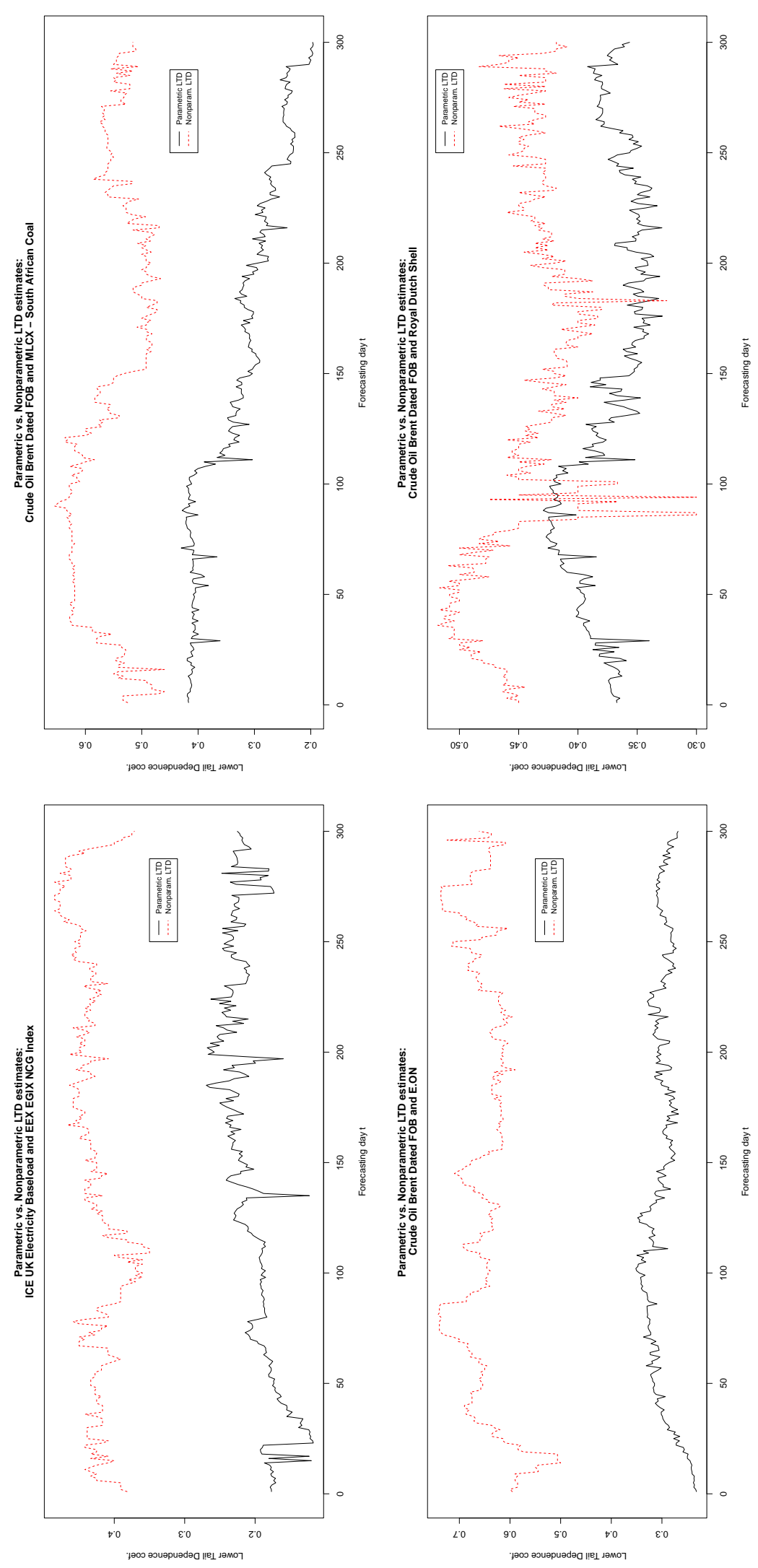

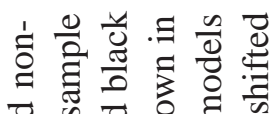

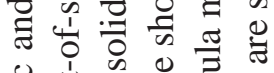

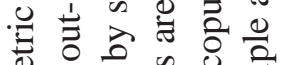
छ

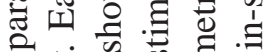

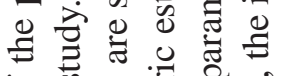
पै 0 \& 0 ह

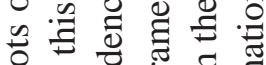

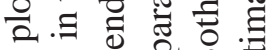

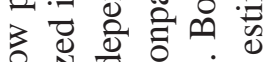
क त्ञ 己

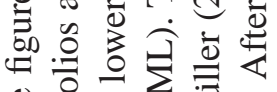
氖氖已完 $\dot{2}$ पे

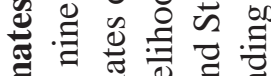
. क

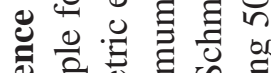

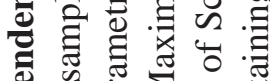
需范

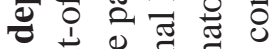

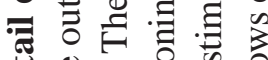
¿ ‡

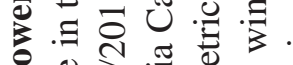
원

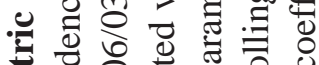

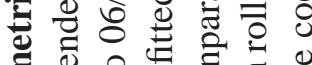

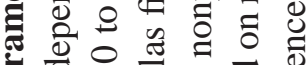
ส음

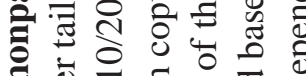
宁

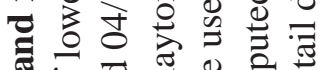

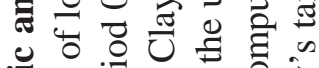

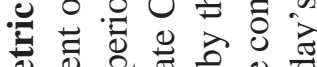

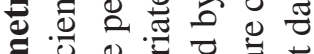
छ

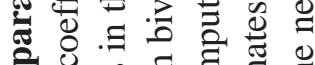

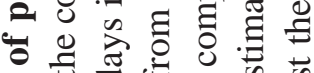
苛 \%

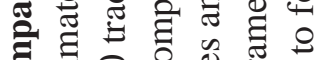
히용

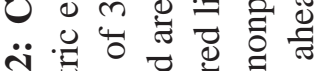

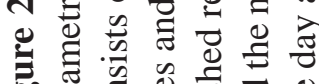

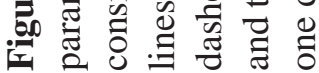



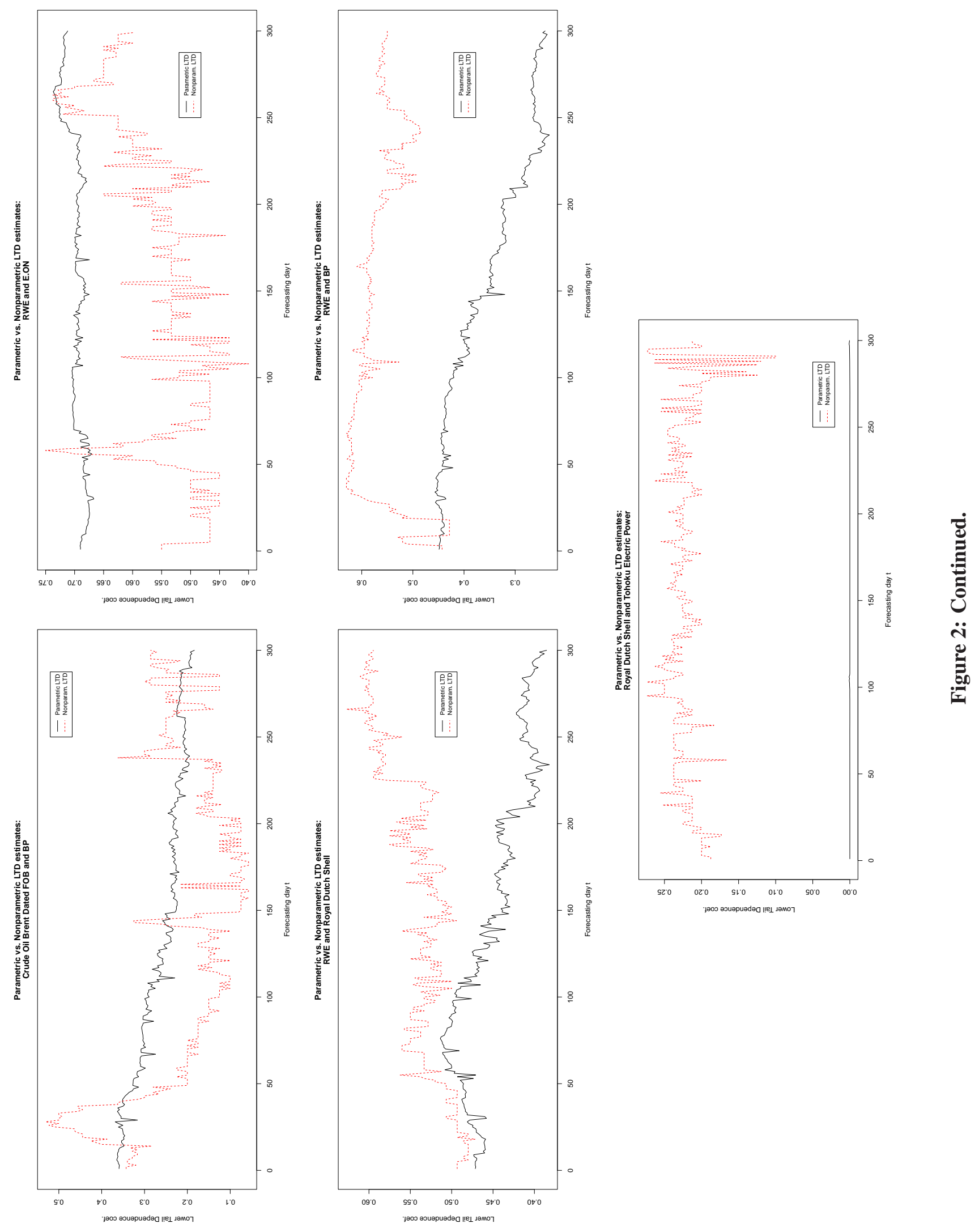

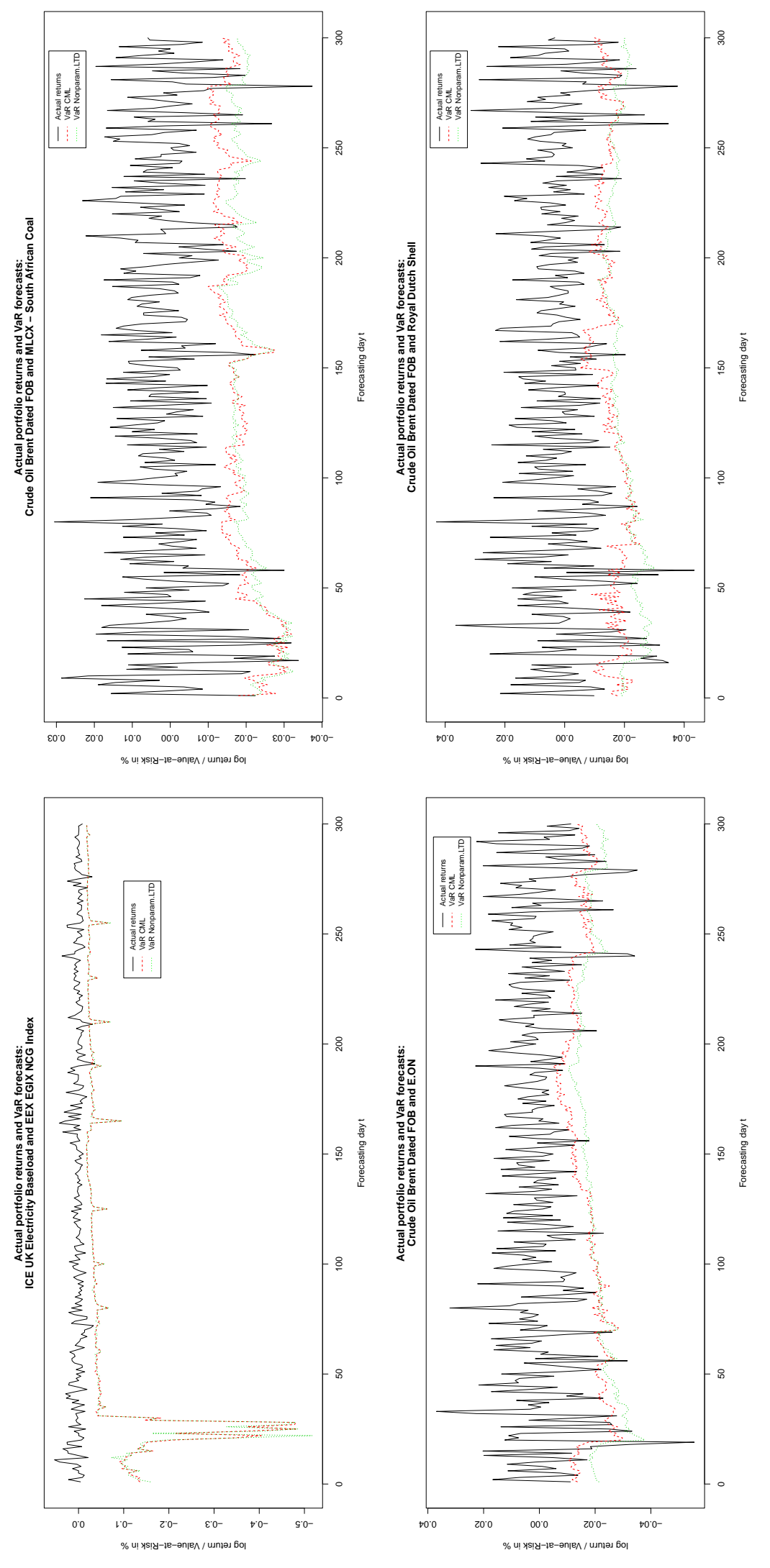

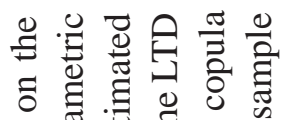

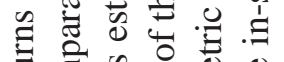

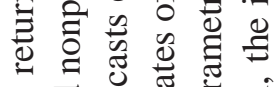

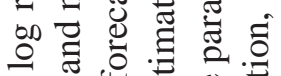

उ.

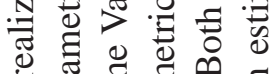

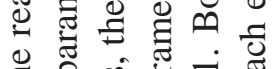

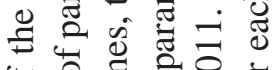

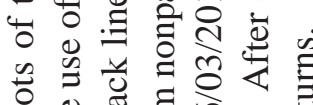

음

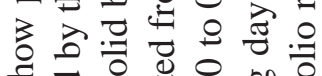

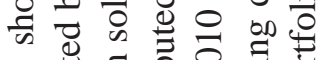

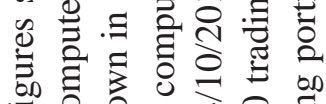

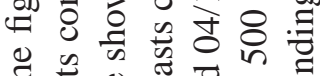

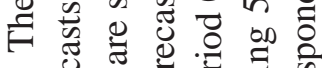

$\dot{0}$ 递它

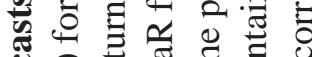

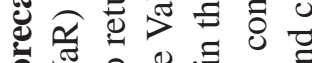

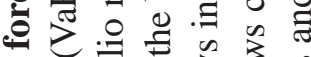

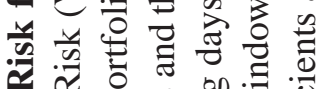

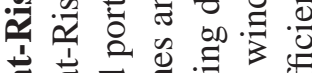

\%

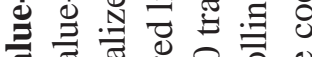

సٓ ॠ

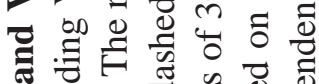

ส

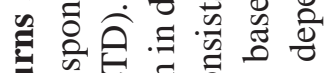

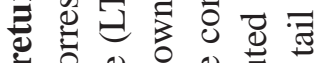

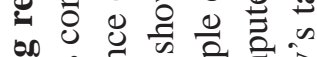

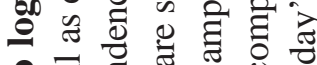

궁

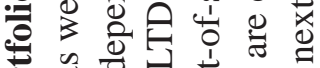

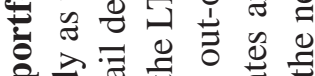

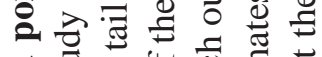

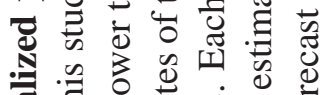

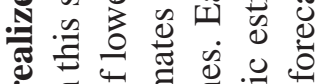

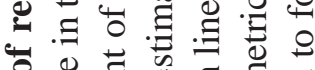

\% 它

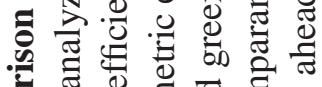

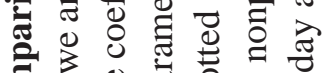

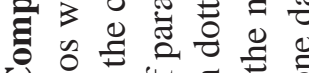

U형

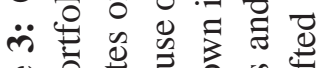

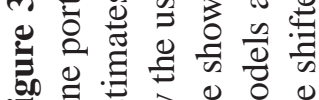

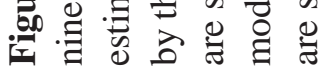



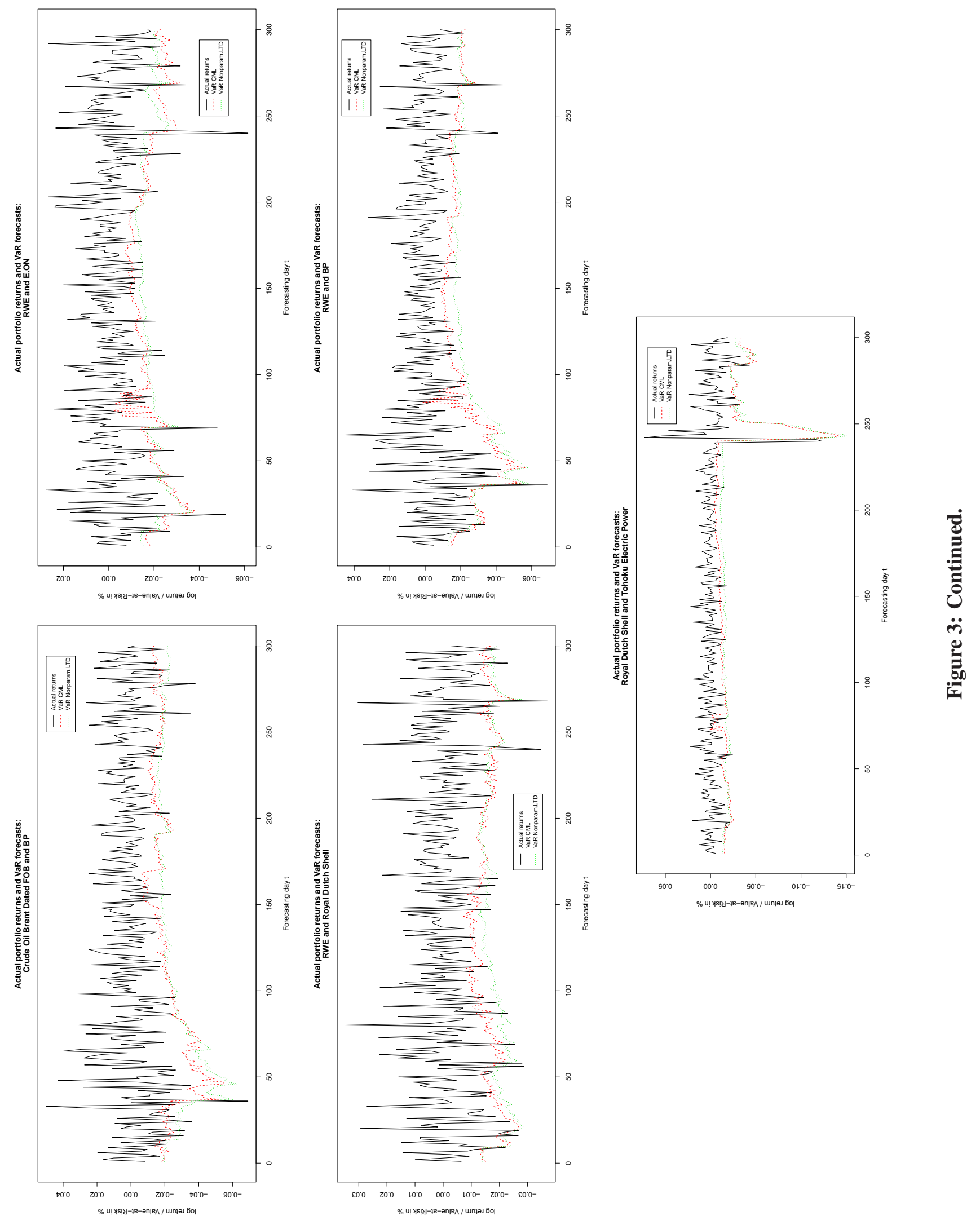


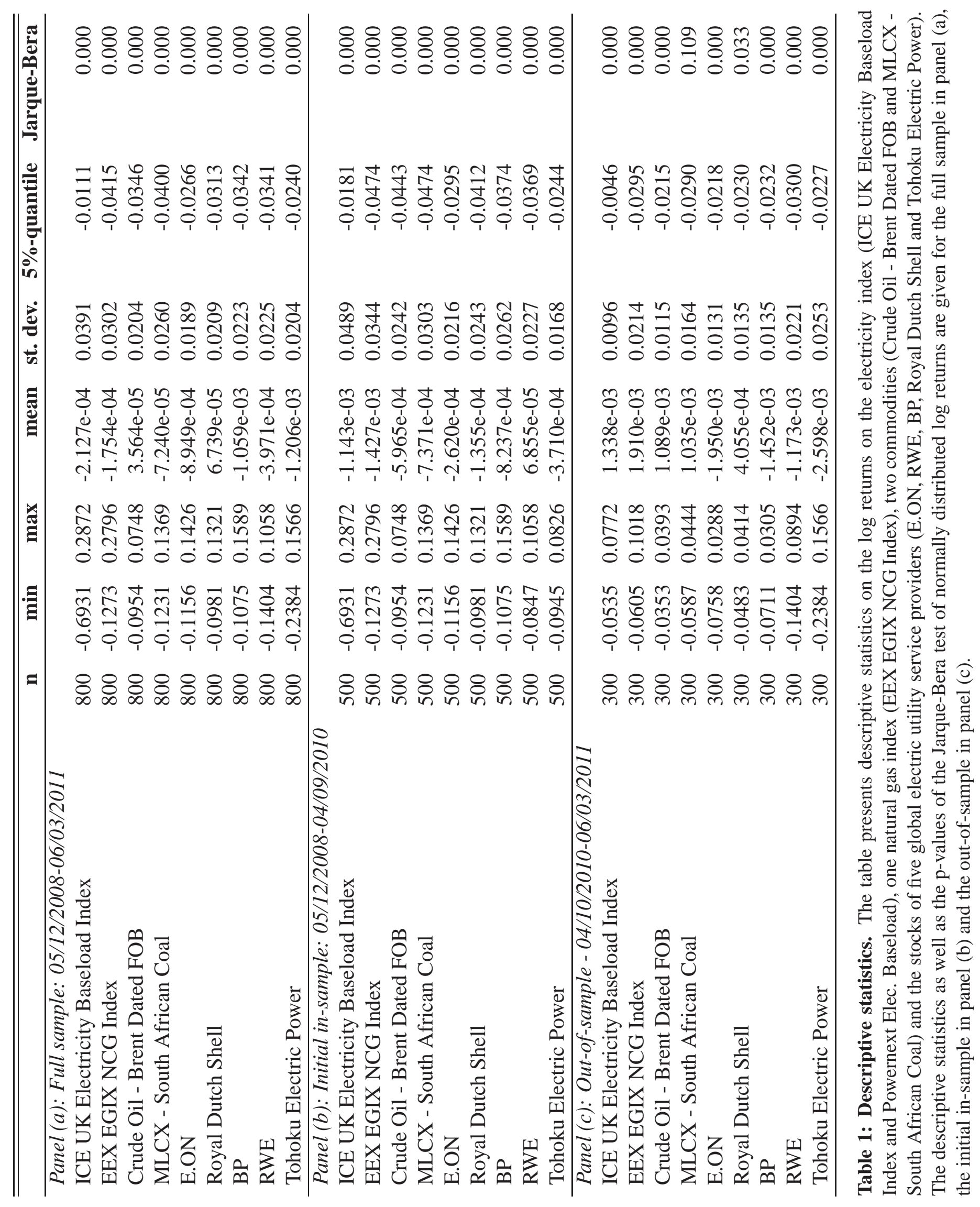




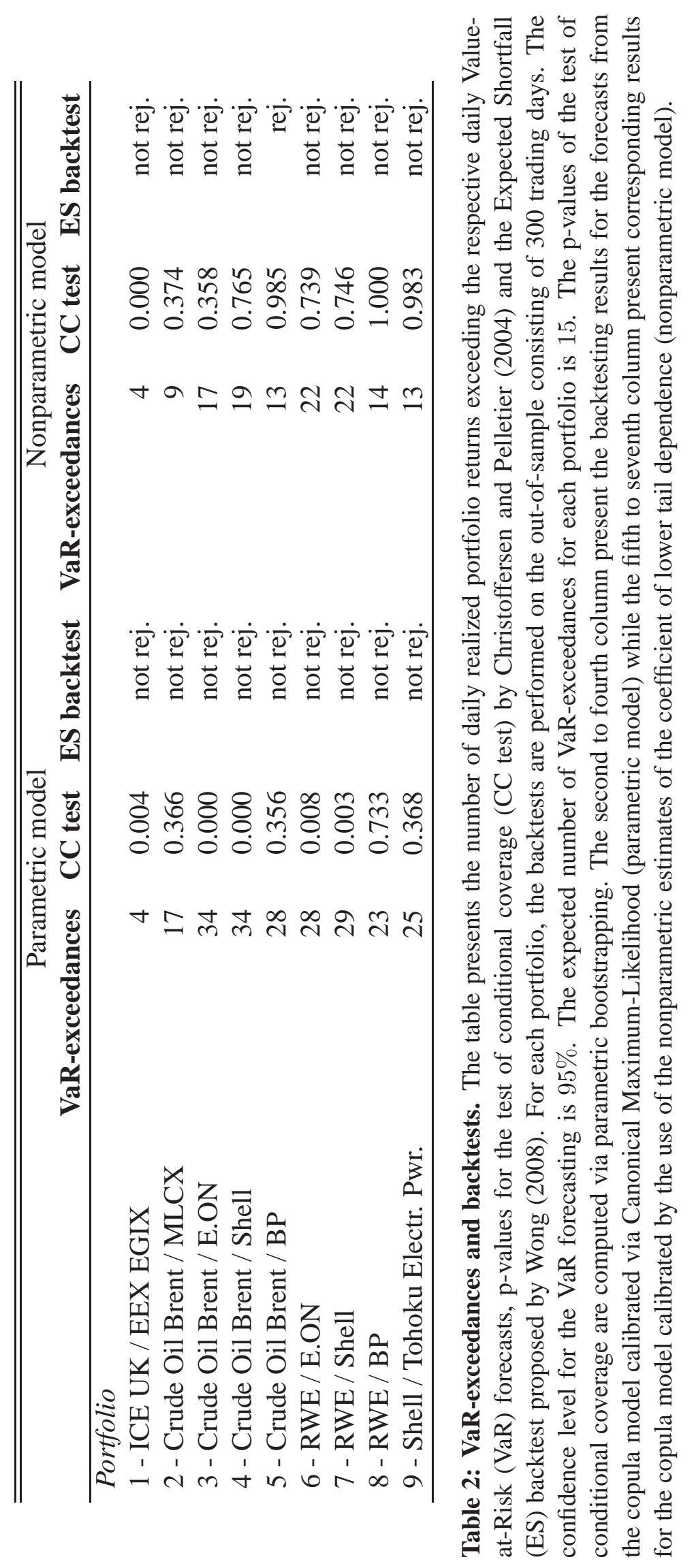




\section{Preprints ab 2011/02}

2013-04

2013-03

2013-02

2013-01

2012-19

2012-18

2012-17

2012-16

2012-15

2012-14

2012-13

2012-12

$2012-11$

2012-10

2012-09

2012-08

Karl Friedrich Siburg, Pavel Stoimenov, and Gregor N.F. Weiß

Forecasting Portfolio-Value-at-Risk with Nonparametric Lower Tail Dependence Estimates

Martin Heida

ON THERMODYNAMICS OF FLUID INTERFACES

\section{Martin Heida \\ EXISTENCE OF SOLUTIONS FOR TWO TYPES OF GENERALIZED \\ VERSIONS OF THE CAHN-HILLIARD EQUATION}

\section{T. Dohnal, A. Lamacz, B. Schweizer}

Dispersive effective equations for waves in heterogeneous media on large time scales

\section{Martin Heida}

On gradient flows of nonconvex functionals in Hilbert spaces with

Riemannian metric and application to Cahn-Hilliard equations

R.V. Kohn, J. Lu, B. Schweizer, and M.I. Weinstein

A variational perspective on cloaking by anomalous localized resonance

Margit Rösler and Michael Voit

Olshanski spherical functions for infinite dimensional motion groups of fixed rank

\section{Selim Esedō̄lu, Andreas Rätz, Matthias Röger}

Colliding Interfaces in Old and New Diffuse-interface Approximations of Willmore-flow

\section{Patrick Henning, Mario Ohlberger and Ben Schweizer}

An adaptive multiscale finite element method

\section{Andreas Knauf, Frank Schulz, Karl Friedrich Siburg}

Positive topological entropy for multi-bump magnetic fields

Margit Rösler, Tom Koornwinder, and Michael Voit

Limit transition between hypergeometric functions of type BC and Type A

\section{Alexander Schnurr}

Generalization of the Blumenthal-Getoor Index to the Class of Homogeneous

Diffusions with Jumps and some Applications

\section{Wilfried Hazod}

Remarks on pseudo stable laws on contractible groups

\section{Waldemar Grundmann}

Limit theorems for radial random walks on Euclidean spaces of high dimensions

\section{Martin Heida}

A two-scale model of two-phase flow in porous media ranging from porespace to the macro scale

\section{Martin Heida}

On the derivation of thermodynamically consistent boundary

conditions for the Cahn-Hilliard-Navier-Stokes system

Michael Voit

Uniform oscillatory behavior of spherical functions of $G L_{n} / U_{n}$ at the identity and a central limit theorem 
Ein optimiertes Glättungsverfahren motiviert durch eine technische Fragestellung

Andreas Rätz

2012-02

A new diffuse-interface model for step flow in epitaxial growth

Hysteresis models and gravity fingering in porous media

$2011-14$

2011-13

$2011-12$

2011-11

$2011-10$

2011-09

2011-08

2011-07

2011-06

2011-05

2011-04

2011-03

\section{Wilfried Hazod}

Intrinsic topologies on H-contraction groups with applications to semistability

\section{Guy Bouchitté and Ben Schweizer}

Plasmonic waves allow perfect transmission through sub-wavelength metallic gratings

Waldemar Grundmann

Moment functions and Central Limit Theorem for Jacobi hypergroups on $[0, \infty[$

\section{J. Koch, A. Rätz, and B. Schweizer}

Two-phase flow equations with a dynamic capillary pressure

\section{Michael Voit}

Central limit theorems for hyperbolic spaces and Jacobi processes on $[0, \infty[$

Ben Schweizer

The Richards equation with hysteresis and degenerate capillary pressure

Andreas Rätz and Matthias Röger

Turing instabilities in a mathematical model for signaling networks

Matthias Röger and Reiner Schätzle

Control of the isoperimetric deficit by the Willmore deficit

Frank Klinker

Generalized duality for k-forms

Sebastian Aland, Andreas Rätz, Matthias Röger, and Axel Voigt

Buckling instability of viral capsides - a continuum approach

\section{Wilfried Hazod}

The concentration function problem for locally compact groups revisited: Non-dissipating space-time random walks, $\tau$-decomposable laws and their continuous time analogues

\section{Wilfried Hazod, Katrin Kosfeld}

Multiple decomposability of probabilities on contractible locally compact groups

\section{Alexandra Monzner* and Frol Zapolsky†}

A comparison of symplectic homogenization and Calabi quasi-states

Stefan Jäschke, Karl Friedrich Siburg and Pavel A. Stoimenov

Modelling dependence of extreme events in energy markets using tail copulas 